\title{
Criminologie
}

\section{Le droit de vote des détenu-e-s}

\section{Jean Claude Bernheim et Renée Millette}

Volume 24, numéro 1, 1991

Les droits des détenu-e-s

URI : https://id.erudit.org/iderudit/017301ar

DOI : https://doi.org/10.7202/017301ar

Aller au sommaire du numéro

Éditeur(s)

Les Presses de l'Université de Montréal

ISSN

0316-0041 (imprimé)

1492-1367 (numérique)

Découvrir la revue

Citer cet article

Bernheim, J. C. \& Millette, R. (1991). Le droit de vote des détenu-e-s. Criminologie, 24(1), 33-48. https://doi.org/10.7202/017301ar
Résumé de l'article

The demand for the right of prisoners to vote has a history that shows how there has never been a right, a freedom, that was recognized without a social, political and legal fight. Rights and freedoms that are not won and whose exercice is not assured remain but words, perhaps merely dreams. d'utilisation que vous pouvez consulter en ligne.

https://apropos.erudit.org/fr/usagers/politique-dutilisation/ 
The demand for the right of prisoners to vote has a history that shows how there has never been a right, a freedom, that was recognized without a social, political and legal fight. Rights and freedoms that are not won and whose exercice is not assured remain but words, perhaps merely dreams.

\section{INTRODUCTION}

Le droit de vote est certainement le droit politique le plus fondamental pour tout individu dans une société libre et démocratique. Il est la pierre angulaire de l'expression et du respect de tous ses droits. C'est pourquoi pour l'Office des droits des détenu-e-s (ODD), la reconnaissance et le respect du droit de vote des détenu-e-s représentent, depuis le début de son action, un objectif majeur, cette reconnaissance équivalant également à la reconnaissance pour le détenu du statut de citoyen à part entière.

L'interdiction du droit de voter imposée aux détenu-e-s est un reliquat de la mort civile qui a prévalu jusqu'en 1906 et de la dégradation civique qui lui a êté substituće jusqu'en 1971. Encore aujourd'hui, certains estiment qu'un contrevenant a décidé, par son geste, de se soustraire à la Charte canadienne des droits et libertés; d'autres pensent que le seul fait d'être en prison devrait priver le condamné de ses droits civils. Que les personnes condamnées n’aient plus aucun droit ni aucune liberté semble être acceptable aux yeux de plusieurs. En effet, pour certains, cette négation de droits est un moyen d'assurer la sécurité du public en maintenant des personnes enfermées; pour d'autres, la prison est un moyen de vengeance.

Plus de dix années de lutte auront été nécessaires pour obtenir un premier jugement confirmant indubitablement notre objectif. L'histoire de cette revendication illustre combien la lutte pour la reconnaissance et le respect des droits et libertés nécessite persévérance et acharnement. Elle illustre aussi le fait qu'il est possible d'avoir gain de cause même quand les victimes du non-respect d'un droit sont des personnes incarcérées, c'est-à-dire des personnes peu sympathiques à l'opinion publique. Elle démontre également que les détenteurs

* Coordonnateur, Office des droits des détenu-e-s, 2120, rue Sherbrooke Est, bureau 215, Montréal (Québec) H2K 1C3

** Avocate, Office des droits des détenu-e-s, 2120, rue Sherbrooke Est, bureau 215, Montréal (Québec) H2K 1C3 
du pouvoir politique s'opposent avec acharnement à la reconnaissance d'un droit et interfèrent, quand cela leur est possible, dans l'exercice de ce droit en dépit de fait qu'il a été reconnu par un tribunal compétent.

\section{LA RECONNAISSANCE DU DROIT DE VOTE DES DÉTENU-E-S AU QUÉBEC}

De 1973 à l' introduction, en 1979, des dispositions prévoyant le droit de vote des détenu-e-s dans la loi électorale du Québec

Au Québec, les premières démarches en vue de faire reconnaître le droit et l'exercice du droit de vote des personnes incarcérées, les prévenus tout comme les condamnés, furent entreprises par l'Office des droits des détenu-e-s. Dès 1973, l'ODD écrivait au président-directeur général des élections du Québec afin de lui demander de faire respecter le droit de vote des personnes détenues en attente de procès, lesquelles, selon nos règles de droit, jouissent de la présomption d'innocence et conservent tous leurs droits, et d'établir un mécanisme qui leur permette d'exercer leur droit de vote dans les établissements de détention. Dans un second temps, l'ODD demandait que soient reconnus le droit de vote et son exercice à toutes les personnes détenues à la suite d'une condamnation judiciaire et recommandait d'amender la loi électorale provinciale en ce sens.

Ainsi débutait une kyrielle de démarches et de pressions de la part de l'ODD et d'autres organismes, comme la Commission des droits de la personne du Québec, afin de faire reconnaître le droit de vote et l'exercice de ce droit pour toute personne incarcérée.

Tout au long de ses démarches, l'ODD s'adressa aux deux niveaux de gouvernement et à la population en général, par voie de lettres, de rapports, de représentations auprès de comités ou devant des commissions parlementaires, de conférences de presse, etc., et collabora étroitement à certains recours judiciaires.

À cette époque, la situation juridique des personnes prévenues, c'est-à-dire en attente de procès, eu égard au droit de vote, était la suivante: aucune loi, fédérale ou provinciale, ne leur retirait expressément ou implicitement le droit de vote. Par contre, aucune loi ne prévoyait de mécanisme permettant l'exercice de leur droit de vote. En ce qui concerne les personnes détenues sous le coup d'une condamnation judiciaire, la Loi électorale du Canada ${ }^{1}$ prévoyait, et prévoit toujours, que :

1. L. R. O. 1985 , c. E-2. 
14(4) Les individus suivants sont inhabiles à voter à une élection et ne doivent voter à une élection:

e) toute personne détenue dans un établissement pénitentiaire (prisons provinciales ou pénitenciers fédéraux) et y purgeant une peine pour avoir commis quelque infraction.

C'est dans l'Acte du cens électoral de $1898^{2}$ qu'est apparue l'interdiction ou l'inhabilité à voter lors d'une élection. Il est évident que le retrait du droit de vote aux prisonniers visait à punir, ce qui est conforme aux objectifs et à la philosophie même de l'incarcération à l'époque.

L'article 6, paragraphe 4, se libellait comme suit:

4. (...) tout individu qui, lors d'une élection, sera incarcéré comme prisonnier dans une geôle ou prison pour y subir la punition de quelque acte criminel, ou qui sera interné dans un asile d'aliénés, ou qui sera entretenu totalement ou partiellement comme pensionnaire nourri et logé dans une maison des pauvres ou un hospice supporté par la municipalité, ou dans une institution de charité recevant l'aide du gouvernement de la province, sera privé du droit de vote et inhabile à voter à aucune élection.

Au Québec, la première négation du droit de vote pour les personnes détenues est inscrite en 1938, dans la Loi électorale du Québec ${ }^{3}$, à l'article 13, dans les mêmes termes que ceux que l'on retrouvait toujours en 1973.

48(i)(d) Ne peuvent être inscrites sur la liste électorale ni voter: les personnes qu'un tribunal compétent a reconnues coupables d'une infraction ou d'un acte criminel punissable de deux ans d'emprisonnement ou plus et qui n'ont pas entièrement purgé la peine prononcée contre elles ${ }^{4}$.

Ainsi, les prévenus et les personnes condamnées pour des délits punissables de moins de deux ans d'emprisonnement conservaient toujours leur droit de vote.

Lorsque l'ODD s'adressait au président-directeur général des élections du Québec ou du Canada, au Comité fédéral des privilèges et des élections, aux divers ministres et députés impliqués dans le débat, tant au pouvoir que dans l'opposition, aux premiers ministres respectifs, il leur rappelait, entre autres :

2. $61 \mathrm{~V}$ chap. 14 , art. 6 , par. 4 .

3. S.R.Q. 1964 , c. 7 , modifiée S.Q. 1965 , c. 12 et 13 ; S.Q. 1966 , c. 5 ; S.Q. 1966-1967, c. 16; S.Q. 1968, c. 11; L.Q. 1969, c. 13 et 19; L.Q. 1971, c. 9; L.Q. 1972, p. 6.

4. 1, Ed. VIII ( $2^{e}$ session) chap. 8, Loi concernant l'élection des députés à l'Assemblée législative. 
— que la mort civile ${ }^{5}$ avait été abolie en $1906^{6}$;

- que la dégradation civique ${ }^{7}$, qui avait remplacé la mort civile, avait été abolie en $1971^{8}$ et que le Code civil avait été amendé en conséquence;

- que depuis plusieurs années, le but et la philosophie des systèmes d'incarcération canadiens n'étaient plus la punition, mais au contraire qu'ils avaient pour objectifs la réhabilitation et la réinsertion sociale des individus qui devraient retourner un jour dans la société, et que retirer le droit de vote à cette catégorie de citoyens était incompatible avec cette idéologie.

Le 19 juillet 1976, dans une lettre qu'adressait l'ODD au directeur général des élections du Canada, M. Jean-Marc Hamel, l'office rappelait à ce dernier, entre autres, le fait que les 160 prisonniers du Centre régional correctionnel de Lower Mainiand (Oakalla) à Burnaby, près de Vancouver en ColombieBritannique, avaient pu voter dans leur institution lors de l'élection provinciale de décembre 1975. La plupart étaient des prévenus, les autres ne purgeaient que de très courtes sentences pour des délits mineurs. L'ODD s'exprimait ainsi:

À moins d'une semaine d'avis, le Procureur Général a organisé la votation avec la collaboration du personnel du service Pre-trial Services and Justice Council et des responsables d'élections. Il y a bien eu des réticences de la part de quelques fonctionnaires, mais cela n'a pas empêché les prisonniers d'Oakalla d'exercer leur droit de vote tel que le veut la loi. Nous croyons qu'il est grandement temps que de telles initiatives se prennent concernant les détenus du Québec.

Le 26 juillet 1976, l'ODD recevait une réponse de M. Hamel énonçant sa position en la matière:

Le Comité (parlementaire) toutefois, n'a pas jugé opportun d'appuyer le principe du projet de loi de $\mathbf{M}$. MacDonald et n'a donc pas recommandé à la Chambre des Communes que les dispositions actuelles de la Loi électorale soient modifiées.

Quant aux prévenus, M. Hamel considérait que le problème résidait dans la façon de voter. La loi prévoyait que le vote devait se faire dans la circonscription électorale de son domicile au moment de l'incarcération et que, par

5. Sur cette question, voir G. E. Kaiser (1971), «The Inmate as Citizen Improvement and the Loss of Civil Rights in Canada», Queen's Law Journal, vol. 1, p. 208.

6. Loi abolissant la mort civile. 6 Ed. VII, chap. 38, art. 1.

7. Id. 6, art. 4 «La dégradation civique consiste: Dans la privation du droit de vote et d'éligibilité et en général de tous les droits civiques et politiques sous le même contrôle législatif."

8. Loi modifiant de nouveau le Code civil et modifiant la Loi abolissant la mort civile. L.Q. 1971 , chap. 82. 
conséquent, la situation du prévenu ne lui permettait pas de s'y rendre pour voter. La loi électorale canadienne ne prévoyait pas de mécanisme pour que les absents puissent voter, à l'exception des membres des Forces armées et des membres de la fonction publique à l'étranger. $M$. Hamel ne jugeait pas opportun non plus d'étendre aux prévenus les dispositions de la loi touchant les électeurs dans les hôpitaux pour malades chroniques ou les foyers pour personnes âgées, ce qui aurait signifié qu'il considérait les institutions de détention comme des lieux de résidence. Il ne voyait que les dispositions du vote par procuration comme pouvant être modifiées afin de s'appliquer aux prévenus, mais il considérait qu'il ne pouvait faire de recommandation en ce sens puisqu'il ne s'agissait ni d'un problème d'ordre technique, ni d'un problème d'ordre administratif.

Au niveau provincial, en novembre 1977 , lors de la Commission permanente de la présidence du Conseil de la constitution et des affaires intergouvernementales concernant le projet de Loi sur la consultation populaire, l'ODD, ainsi que d'autres groupes, fit des représentations visant précisément à faire reconnaître le droit de vote à toute personne incarcérée, tant dans les établissements de détention fédéraux que provinciaux.

L'ODD, pour sa part, concluait que les personnes incarcérées, malgré l'interdiction de circuler qui les frappe, n'en demeurent pas moins des citoyens à part entière, et faisait les recommandations suivantes ${ }^{9}$ :

1) Que toute personne incarcérée au Québec ne soit pas privée de son droit de vote du seul fait de son incarcération;

2) Que ce droit puisse être exercé:

a) en considérant comme domicile de la personne l'endroit où elle habitait avant son incarcération;

b) en considérant comme domicile de la personne la maison de détention où elle est gardée; le choix d'une des deux alternatives devant être fait par le citoyen incarcéré lui-même;

3) Que dans tout établissement de détention (provincial ou fédéral) se tiennent des bureaux de votation avancés, que les bulletins soient redistribués dans les comtés respectifs;

4) Que les gouvernements apportent des changements à la loi électorale et à toutes les lois provinciales (exemples: loi des cités et villes, code municipal, chartes spéciales, etc.) traitant de la tenue du scrutin en

9. Mémoire de l'ODD présenté le 17 novembre 1977. L'intervention de l'ODD se trouve dans Québec, Assemblée nationale, Commission permanente de la présidence du Conseil, de la Constitution et des Affaires intergouvernementales, $31^{\mathrm{e}}$ législature, $2^{\mathrm{e}}$ session, Étude du livre blanc sur la consultation populaire au Québec. 
vue de rendre celles-ci conformes à la loi de la consultation populaire en ce qui concerne le droit de vote des personnes incarcérées;

5) Que la loi de la consultation populaire ne discrimine pas les individus en raison de leur casier judiciaire (i.e. que la possession d'un casier judiciaire ne soit pas un motif pour interdire à une personne l'accès au poste d'officier d'élection);

6) Qu'il y ait des amendements aux lois actuelles (loi électorale, loi des cités et villes, etc.) afin d'éliminer la discrimination fondée sur le casier judiciaire.

À la suite de l'étude en commission parlementaire du projet de Loi sur la consultation populaire, le gouvernement québécois proposa le projet de loi 92. L'ODD revint à la charge en faisant remarquer au ministre responsable que des lacunes subsistaient toujours dans le projet, soit celle qui concernait les détenus déclarés coupables pour des infractions punissables de deux ans et plus, qui n'avaient toujours pas le droit de voter à une élection provinciale ${ }^{10}$, et celle relative à la persistance dans le projet de loi de restrictions concernant les officiers d'élections. Le 15 mai 1978, le ministre Burns informa l'ODD de la création d'un comité ministériel composé de députés et de membres de son cabinet afin de faire une révision complète de la loi électorale et d'y apporter les amendements nécessaires. En effet, comme l'adoption de la Loi sur la consultation populaire avait rendu nécessaire une réforme de la loi électorale québécoise et de ses lois connexes, le comité Dussault avait été formé à cette fin. Il étudia, entre autres, la question du droit de vote des personnes incarcérées, tant les prévenus que les individus sous le coup de sentences dans les établissements fédéraux ou provinciaux.

Le principe moteur qui guida le comité Dussault tout au long de son étude sur la question du droit de vote en général fut de faire de la primauté absolue de l'électeur le principe premier du projet de loi 9, ou Loi électorale. D'ailleurs, le parrain de ce projet de loi, le ministre de la Justice de l'époque, Me MarcAndré Bédard, déclarait, concernant le droit de vote et le droit de candidature en général, qu'ils «constituent l'assise première des mécanismes de représentation politique et c'est par eux que peut se mesurer le chemin parcouru sur le plan de l'accession à une société authentiquement démocratique ${ }^{11}$.

Le comité Dussault déposa son rapport en juin 1978. Dans ses conclusions concernant le droit de vote des personnes détenues, il étendait à tous les pri-

10. Dans sa définition de l'électeur, Ja Loi sur la consultation populaire, L.Q. (1978) c. 6, référait à la Loi électorale, S.R.Q. 1964 c. 7 telle qu'amendée, où l'article 48 (d) prévoyait spécifiquement que les détenus déclarés coupables d'infractions punissables de deux ans et plus ne pouvaient voter.

11. Journal des débats, Assemblée nationale, Session 1979, vol. 21, n 59, p. 3237. 
sonniers et prisonnières le droit de vote. M. Dussault déclara à l'Assemblée nationale, lors du dépôt en deuxième lecture du projet de loi : «Cette réforme traduit une volonté réelle et pleinement réfléchie de favoriser encore davantage les possibilités de réhabilitation de ces citoyens ${ }^{12}$.»

À sa suite, des députés de l'opposition comme M. Harry Blank affirmèrent que personne ne pouvait être contre ${ }^{13}$, d'autres, comme $M$. Jean-Noël Lavoie, dirent que le droit de vote devrait être libéralisé le plus possible de manière qu'il y ait le moins de contraintes possibles. Il fallait que le fardeau de la preuve ne repose pas sur l'électeur, qu'il y ait une preuve prima facie, un droit à l'exercice du droit de vote ${ }^{14}$.

Finalement, le 13 décembre 1979 , la Loi électorale du $Q u e ́ b e{ }^{15}$ fut sanctionnée; elle prévoyait des dispositions particulières concernant le droit de vote des personnes détenues et son exercice.

La loi provinciale établissait, sans ambiguïté, le droit de vote lors de toute élection provinciale de toute personne détenue dans des établissements de détention établis par une loi du Parlement du Canada ou par la Législature, ainsi qu'un mécanisme précis pour exercer ce droit et une possibilité pour le directeur général des élections du Québec de conclure toute entente qu'il jugerait utile avec des directeurs des établissements de détention, tant fédéraux que provinciaux, afin de permettre et de faciliter l'exercice du droit de vote des personnes incarcérées ${ }^{16}$.

Le Québec établissait donc un précédent législatif pour tout le Canada. Au cours des élections provinciales québécoises de 1981, 1985 et 1989, les détenus sous juridiction provinciale, incarcérés dans une prison provinciale, purent exercer leur droit de vote sans aucun problème.

\section{Référendum québécois du 20 mai 1980}

À la suite de l'adoption de la Loi sur la consultation populaire et de la réforme de la Loi électorale du Québec, tous les détenus du Québec, tant des pénitenciers fédéraux que des prisons provinciales, ont pu, pour la première fois, exercer leur droit de vote au cours du référendum du 20 mai 1980.

D'ailleurs, le Solliciteur général du Canada de l'époque, M. Robert Kaplan, déclarait, le 15 juillet 1980 , devant le comité Justice et questions juridiques de la Chambre des communes: «Cette expérience (le référendum) nous

12. Ibid., p. 3239.

13. Commission parlementaire, Sess. 1979 , vol. $21, \mathrm{n}^{\circ} 202$, p. B-100.23.

14. Voir supra, note 11, p. 245.

15. L.Q. (1979) c. 56.

16. Ibid., art. 64 . 
a donc appris qu'il est possible de permettre aux détenus de participer à une élection ${ }^{17}$."

À la suite de cet événement qui s'est déroulé dans l'ordre et sans incident, les détenus étaient en droit de croire que leur droit de vote était officiellement établi et reconnu.

\section{Élections générales provinciales québécoises du 13 avril 1981}

Bien que la Loi électorale du Québec ait été amendée et que tous les détenus aient pu s'exprimer lors du référendum de 1980, les détenus des pénitenciers fédéraux se sont vu refuser l'exercice de leur droit de vote lors des élections provinciales qui avaient été prévues pour le 13 avril 1981.

En effet, quoique le Solliciteur général du Canada eût déclaré en juillet 1980: «J'ajoute en conclusion que les Nations unies ont promulgué un traité dont le Canada est signataire, qui prévoit l'attribution du droit de vote. Nous n'avons donc pas respecté cette obligation internationale ${ }^{18}$, celui-ci refusa de signer le protocole d'entente qui aurait permis aux détenus sous juridiction fédérale d'exercer leur droit de vote. En conséquence, l'ODD prenait l'initiative d'une «requête pour l'émission d'une injonction provisoire et interlocutoire ${ }^{19}$ " qui aurait permis aux détenus des pénitenciers fédéraux du Québec, si elle avait été accordée, d'exercer leur droit de vote. Mais le juge Marceau, dans son jugement du 30 mars 1981 , tout en admettant que «ce n'est pas la reconnaissance du droit de vote lui-même qui est en cause", estimait qu'en l'espèce, il n'était pas déraisonnable de priver les détenus des pénitenciers de l'exercice de leur droit de vote du fait de leur incarcération ${ }^{20}$.

La situation dominante en droit canadien avant l'avènement de la Charte canadienne des droits et libertés était la suivante: même si les tribunaux avaient graduellement reconnu l'existence d'un droit à un ou plusieurs individus, s'ils étaient convaincus que la Couronne ou ses représentants leur retiraient ce droit en vertu d'une loi du Parlement dans le cadre de l'exercice de leurs fonctions, alors la Cour ne pouvait garantir l'exercice ou la protection de ce droit.

17. Canada, Chambre des communes, Comité permanent de la justice et des affaires juridiques, $32^{\mathrm{e}}$ législature, $1^{\text {re }}$ session, procès-verbaux et témoignages, fasc. 5, p. 61,15 juillet 1980.

18. Ibid.

19. Lévesque et al. c. Kaplan, (1985) 1 O.F. 496.

20. Ibid. 


\section{Communication au Comité des droits de l'homme R. 25/113}

Tout recours en appel étant illusoire, compte tenu des délais et de la tenue des élections le 13 avril, il fut décidé d'adresser une communication au Comité des droits de l'homme des Nations unies, organe constitué par la quatrième partie du Pacte international relatif aux droits civils et politiques et agissant en application du protocole facultatif se rapportant à ce Pacte. Cette communication fut déposée directement à Genève par les représentants de la Fédération internationale des droits de l'homme, le 10 décembre 1981.

Après avoir pris connaissance de la «Réponse du Canada à la question de ja recevabilité de la communication ${ }^{2 l}$ » et des «Commentaires de $\mathrm{C}$. Forget et al. concernant la réponse du Canada ${ }^{22}$, le Comité des droits de l'homme notait dans sa décision sur la recevabilité, le 23 juillet 1983 :

De toute façon le gouvernement canadien n'a pu jusqu'à ce jour démontrer dans l'affaire à l'examen qu'une action en jugement déclaratoire aurait constitué un recours utile en ce qui concerne soit les élections du 13 avril 1981 , soit des élections futures. Si l'on se réfere aux observations du gouvernement en date du 20 août 1982, il n'apparaît pas clairement que les autorités auraient jugé recevable une action dont l'objet était d'obtenir que soit déclaré illégal le refus des autorités pénitentiaires compétentes de permettre aux victimes présumées de participer aux élections du 13 avril 1981. D'autre part, compte tenu des observations reçues de l'auteur le 7 juin 1983, on peut sérieusement se demander si les autorités exécutives au Canada seraient tant soit peu tenues de donner suite à un jugement déclaratoire au cas où une situation analogue se reproduirait à l'avenir, et jusqu'à quel point elles le seraient.

Il concluait sa décision dans les termes suivants:

1. Que la communication est recevable;

2. Que, conformément au paragraphe $\mathbf{2}$ de l'article $4 \mathrm{du}$ Protocole facultatif, l'État partie sera prié de lui soumettre par écrit, dans les six mois qui suivront la date de la transmission de la présente décision, des explications ou des déclarations éclaircissant la question et indiquant, le cas échéant, les mesures qu'il pourrait avoir prises pour remédier à la situation;

21. Réponse du Canada, daté du 20 août 1982, à la question de la recevabilité de la Communication $n^{\circ}$ R $25 / 113$ présentée par Messieurs Claude Forget, Michel Leblanc et JeanLouis Lévesque, p. 7.

22. Commentaires de Claude Forget et al. concernant la réponse du Canada à la question de recevabilite de la communication $n^{\circ} R$. 25/113, 1982. 
3. Que le Secrétaire général communiquera aux auteurs, conformément au paragraphe 3 de l'article 93 du règlement intérieur provisoire du Comité, toutes les explications ou déclarations reçues de l'État partie, en les priant de faire parvenir tous commentaires qu'ils pourraient souhaiter présenter à leur sujet au Comité des droits de l'homme, par l'entremise du Centre pour les droits de l'homme, Office des Nations Unies à Genève, dans les six semaines qui suivront la date de la transmission;

4. Que la présente décision sera communiquée à l'État partie et aux auteurs de la communication ${ }^{23}$.

Se prévalant de son droit de révision en vertu de l'article 93(4) du Règlement intérieur provisoire, le Canada soutint notamment dans sa «Réponse ${ }^{24}$ » que depuis le dépôt de la communication, en décembre 1981, la Charte canadienne des droits et libertés ${ }^{25}$ avait été adoptée et que les auteurs de la communication n'avaient pas épuisé tous les recours internes disponibles et, par conséquent, qu'ils devaient avoir recours à une requête en jugement déclaratoire. Afin de donner plus de poids à son argumentation, le Canada donnait au Comité des droits de l'homme l'assurance «que si un jugement déclaratoire définitif disant que le Solliciteur général a agi illégalement en ne prenant pas les mesures nécessaires pour que les détenus des pénitenciers fédéraux puissent voter lors de l'élection générale québécoise du 13 avril 1981 venait à être prononcé, il prendrait les mesures en question à l'égard de toute élection générale qui aurait lieu à l'avenir au Québec ${ }^{26}$ ». Finalement, le 12 avril 1985, le Comité des droits de l'homme annulait sa décision antérieure, estimant que «la possibilité d'obtenir un jugement déclaratoire existe ${ }^{27}$ ».

\section{Élections générales provinciales québécoises du 2 décembre 1985}

Dans la perspective de la tenue d'élections provinciales au Québec, le directeur général des élections du Québec écrivait au Solliciteur général du Canada, le 27 mars 1985, pour l'inviter à entamer les démarches préliminaires

23. Comité des droits de l'homme, décision, Communication R. 25/113, Doc. N.U. OOPR/O/19/D/R. 25/113 (1983).

24. «Réponse du Canada à l'invitation que lui a faite le Comité des droits de l'homme de présenter par écrit des explications ou déclarations relativement à la Communication R. 25/113», 17 février 1984, 24 pages.

25. Charte canadienne des droits et libertés, Canada Act 1982, c. 11 (R.U.) Schedule B : Loi constitutionnelle de 1982, Partie I.

26. Comité des droits de l'homme, décision, Communication R. 25/113, Doc. N.U. OOPR/O/24/D/R.25/113 (1985), 12 avril 1985, reproduit dans Rapport au comité des droits de l'homme, Doc. off. A.G., $40^{\circ}$ session, supp. $n^{\circ} 40$, Doc. N.U.A. $/ 40 / 40$, annexe XV, p. 233.

27. «Réponse du Canada...», loc. cit. 
à la signature d'un protocole d'entente qui aurait assuré aux détenus des pénitenciers fédéraux du Québec l'exercice de leur droit de vote.

Devant le mutisme des autorités fédérales, l'ODD décida de s'adresser encore une fois à la Cour fédérale du Canada afin d'obtenir un jugement déclaratoire, ainsi que le suggérait le Comité des droits de l'homme. Parallèlement à cette première requête en jugement déclaratoire, était déposée une «requête afin d'obtenir dispense des règles de la Cour fédérale en matière d'une action visant à obtenir un jugement déclaratoire et pour une audition au mérite d'urgence».

Dans un premier jugement concernant la deuxième requête, le juge P. Rouleau ordonnait aux parties d'agir avec célérité, compte tenu de la proximité des élections provinciales fixées au 2 décembre 1985, tout en accordant des délais suffisants pour assurer aux ministres intéressés une défense pleine et entière. C'est ainsi que les parties devaient se présenter devant le tribunal le 22 novembre pour que fût fixée une date d'audition. Celle du 26 novembre fut retenue. Le procès ayant eu cours toute la journée du 26 novembre, le juge rendait sa décision ${ }^{28}$.

Ce jugement peut être qualifié d'historique en ce qu'il reconnaît sans ambages le statut de citoyens à part entière aux détenus, sans égard à leur incarcération, et le droit de participer à la vie politique du pays et des provinces par l'exercice du «droit de vote (qui) est la pierre angulaire de toute démocratie qui se respecte», comme le souligne le juge dans son jugement. Cette reconnaissance d'un droit fondamental aux détenus par un tribunal est extrêmement importante, eu égard à l'objectif de l'ODD dans sa lutte pour la reconnaissance et le respect des droits des détenus. En effet, en 1972, quand l'ODD fut mis sur pied, la notion de droits des détenus était encore inhabituelle, sinon suspecte. Le présent jugernent, contrairement aux autres rendus en d'autres circonstances, ne veille pas seulement au respect des principes de justice qui nous gouvernent, mais reconnaît solennellement un droit politique aux personnes incarcérées.

De plus, ce jugement concerne non seulement les droits des détenus, mais également ceux de tous les Canadiens, parce que, tout d'abord, il énonce clairement que «si la Charte canadienne des droits et libertés, qui fait partie de la Constitution du Canada, est la loi suprême du pays, nul ne peut y échapper, pas même la Couronne ni un ministre agissant en sa qualité de représentant de la Couronne»; et ensuite parce qu'il constate que la «Charte a non seulement modifié le droit existant mais l'a également bouleversé» et que par conséquent

28. Lévesque c. P.G. du Canada, (1985) D.L.R. (4th) 184, (1986) 2 C.F. 287, 20 O.R.R. 15. 
un bref de mandamus peut être émis contre la Couronne ou un de ses représentants.

Ce bref de mandamus a pour effet d'annuler toutes les règles d'immunité issues de la common law, compte tenu du respect de la Charte; par conséquent, «il ne fait plus de doute maintenant que la Couronne est assujettie aux dispositions de la Charte au même titre que tout autre administré».

Il ordonne également que tous les détenus des pénitenciers fédéraux du Québec puissent exercer leur droit de vote s'ils le désirent. Ce que ceux-ci ne se sont pas privés de faire avec tout le sérieux requis. Aux élections provinciales de 1989 , les détenus des pénitenciers fédéraux ont pu voter normalement.

Plus de dix années d'efforts ont été nécessaires pour que finalement le droit de vote des détenus soit reconnu et que l'exercice en soit assuré au niveau provincial au Québec. Même si l'ODD prit l'initiative de plusieurs démarches, il va sans dire que d'autres ont aussi collaboré à cette reconnaissance, soit par leurs prises de position ${ }^{29}$, soit par leurs recours, à faire du Canada, même à l'encontre du gouvernement fédéral, un des pays qui appliquent partiellement l'article 25b) du Pacte international relatif aux droits civils et politiques.

Terre-Neuve a pour sa part reconnu le droit de vote aux détenus en modifiant sa loi électorale en 1985. Le Manitoba ${ }^{30}$, en 1986 , et l'Ontario ${ }^{31}$, en 1988 , ont reconnu aux détenus le droit de vote au niveau provincial après que ceux-ci ont eu gain de cause devant les tribunaux de leur province.

\section{LA NON-RECONNAISSANCE DU DROIT DE VOTE DES DÉTENUS AUX ÉLECTIONS FÉDÉRALES}

En ce qui concerne les démarches à caractère politique concernant le droit de vote des détenus lors d'élections fédérales, elles se sont essentiellement traduites par des échanges de correspondance avec le directeur général des élections, M. Jean-Marc Hamel, le président du Comité des privilèges et élections, différents députés et ministres et, finalement, avec les premiers ministres John Turner et Brian Mulroney. Ces démarches ont été moins nombreuses du fait que notre attention était surtout centrée sur le Québec, où le dossier avançait plus rapidement grâce à l'intérêt favorable manifesté, entre

29. Voir supra, note 9.

30. Badger C. A.-G. of Manitoba (1986) 30 D.L.R. (4th) 108, 27 O.O.O. (3d) 158, 39 Man. R. (2d) 107,51 O.R. (3d) 163, 21 O.R.R 277; conf. 32 D.L.R. (4th) $310,290.0 .0$ (3d) 92, 39 Man. R. (2d) 230, 55 O.R. (3d) 364, 21 O.R.R. 379.

31. Grondin c. A.-G. Ont. (1988) 65 O.R. (2d) 427. 
autres, par la Commission des droits de la personne, le directeur général des élections, M. Pierre-F. Côté, et plusieurs députés.

Il est à noter qu'en 1975, le député fédéral d'Egmont (Île-du-PrinceÉdouard), M. David McDonald, a présenté un projet de loi privé visant à reconnaître le droit de vote aux détenus lors des élections fédérales. Son initiative n'a pas été couronnée de succès.

En 1988, le député fédéral de Burnaby (C.-B.), M. Svend Robinson, a appuyé sans réserve nos démarches auprès des autorités politiques fédérales.

En ce qui concerne le droit de vote et son exercice lors des élections fédérales, plusieurs recours ont été entrepris non seulement au Québec mais aussi en Ontario et au Manitoba.

C'est en 1984 que le Correctional Law Project, de la faculté de droit de l'université Queen, à Kingston, et l'ODD ont pris en charge les premiers recours judiciaires. Robert Gould, du pénitencier de Joyceville (Ontario), et Germain Gagnon, du pénitencier Leclerc (Québec), ont présenté une requête devant la Cour fédérale du Canada, et Richard Sauvé, du pénitencier Collins Bay (Ontario), a fait de même devant la Cour suprême de l'Ontario (équivalent de la Cour supérieure du Québec).

Robert Gould a obtenu, suite à sa requête en injonction, le droit de voter par procuration aux élections fédérales du 4 septembre. Immédiatement après cette décision, le gouvernement du Canada est allé en appel et la Cour d'appel fédérale, dans un jugement partagé, a estimé que le juge de première instance avait statué prématurément sur la requête, n'ayant pas eu l'occasion d'entendre le procès afin d'établir éventuellement le bien-fondé de la requête. La Cour suprême a pour sa part maintenu la décision de la Cour d'appel ${ }^{32}$. Le dossier Gagnon n'a pas été poursuivi.

C'est en septembre 1988 que le juge Mabel Van Camp, de la Cour suprême de l'Ontario, entendait la requête déposée par Richard Sauvé en 1984, à la veille des élections fédérales qui se sont tenues cette année-là. Le 7 novembre, soit plus de quatre ans après le dépôt de la requête, elle rendait une décision estimant que l'interdiction de voter contenue dans la Loi électorale du Canada n'est pas incompatible avec la Charte ${ }^{33}$. Cette décision est devant la Cour d'appel.

32. Gould c. P.G. du Canada (1984) 1 O.F 1119, 42 O.R. (3d) 78; inf. 1 O.F. 1133, 42 O.R. (ed) 88, 13 D.L.R. (4th) 485, 54 N.R. 232; conf. 2 R.O.S. 124, 13 D.L.R. (4th) $491 n$, 53 N.R. $394 n$.

33. Sauvé c. A. -G. of Canada (1988) 53 D.L.R. (4th) 595, 66 O.R. (2d) 234. 
En prévision des élections fédérales en mars 1988, l'ODD soutenait le recours entrepris par Jean-Louis Lévesque, basé sur les mêmes arguments invoqués lors de sa requête antérieure, afin d'obtenir la reconnaissance du droit de vote pour les détenus aux élections fédérales ${ }^{34}$. La volonté de présenter un dossier le plus solide possible a fait en sorte que les élections se sont déroulées avant que le procès n'ait été entendu.

Sans avoir produit de défense, le procureur du gouvernement du Canada a déposé, le 17 janvier 1990, une requête en radiation des plaidoiries «aux motifs que l'action du demandeur ne révèle aucune cause raisonnable d'action ${ }^{35}$ ». Il est pour le moins stupéfiant que, malgré le jugement clair et non équivoque du juge Rouleau et des tribunaux du Manitoba et de l'Ontario, le gouvernement du Canada plaide l'absence de cause raisonnable. La Cour a rejeté cette assertion et la cause devrait normalement suivre son cours.

En septembre également, Arnold Badger, celui-là même qui eut gain de cause relativement à la reconnaissance du droit de vote au niveau provincial au Manitoba, ainsi que deux codétenus du pénitencier Stony Mountain ont déposé une requête pour obtenir la reconnaissance du droit de vote aux élections fédérales du 21 novembre 1988. Le 4 novembre, le juge Hirschfield, de la Cour du banc de la reine, a conclu que l'article 14(4)(e) de la Loi électorale du Canada contrevenait à la Charte, que les détenus avaient le droit de vote et ordonnait que le directeur général des élections agisse en conséquence.

Le gouvernement du Canada est allé en appel et la Cour d'appel du Manitoba a renversé la décision dans un jugement unanime rendu le 18 novembre. Les détenus ont interjeté appel devant la Cour suprême du Canada, mais celle-ci a rejeté la requête en autorisation de pourvoi ${ }^{36}$.

En 1990, la Commission royale sur la réforme électorale et le financement des partis a tenu des audiences à travers le pays et a abordé l'épineuse question du droit de vote des détenus aux élections fédérales. L'ODD a soumis un mémoire et a comparu devant la Commission pour défendre son point de vue ${ }^{37}$. Le rapport de la commission devrait être rendu public incessamment.

Compte tenu de la position du gouvernement fédéral devant le Comité des droits de l'homme des Nations unies, le plus surprenant est que le gou-

34. Sauvé c. A.-G. of Canada (1988) 53 D.L.R. (4th) 595, 66 O.R. (2d) 234. T-531-88.

35. Lévesque c. P. G. du Canada et Solliciteur général du Canada, O.F (1 ${ }^{\mathrm{r}}$ instance),

36. Badger c. A.-G. of Canada (1989) 55 D.L.R. (4th) 177; (1989) I R.O.S.V.

37. Office des droits des détenu-e-s (1990), Le Droit de vote des détenu-e-s, Montréal, ODD, 26 mars 1990, $46 \mathrm{p}$. 
vernement refuse toujours de prendre en compte le jugement Rouleau pour rendre conforme la Loi électorale du Canada aux principes démocratiques qui nous gouvernent et à la Charte canadienne des droits et libertés.

\section{L'EXERCICE DU DROIT DE VOTE}

La situation actuelle peut se résumer comme suit: au Québec, à TerreNeuve, au Manitoba et en Ontario, les détenus des prisons provinciales et des pénitenciers fédéraux se sont vu reconnaître le droit de vote à des élections provinciales, soit par des amendements législatifs, soit par des décisions judiciaires.

Il est à remarquer que l'exercice de ce droit ne va pas sans problème dans certaines provinces. En effet, en Ontario par exemple, l'exercice du droit de vote se fait par procuration, ce qui oblige les détenus à confier à un ami ou un parent le soin de voter à leur place. Et comme plusieurs d'entre eux n'ont pas d'amis, de famille ou ont rompu avec celle-ci, l'exercice de ce droit devient illusoire. Il va donc falloir que des interventions soient entreprises pour obtenir que l'exercice du droit de vote soit garanti de manière équitable. L'exemple du Québec démontre très clairement qu'il est tout à fait possible que des bureaux de scrutin soient installés dans les institutions carcérales.

Au Manitoba, les autorités provinciales ont décidé unilatéralement, en dépit du fait que les détenus ont voté en 1988, de suspendre l'exercice de leur droit de vote lors des élections provinciales du 11 septembre 1990, sous prétexte que la Cour d'appel du Manitoba avait rendu un jugement le 21 novembre 1988 estimant que la Loi électorale du Canada ne contrevient pas à la Charte en retirant le droit de vote aux détenus.

En ce qui concerne la reconnaissance du droit de vote lors des élections fédérales, le gouvernement s'y oppose avec acharnement, et les cours de justice ne l'ont pas encore reconnu, au contraire.

\section{CONCLUSION}

Comme nous l'avons vu, l'obtention du droit de vote pour les détenus était et est toujours, pour l'ODD, une question d'abord politique, la lutte judiciaire représentant un moyen pour obtenir la reconnaissance et l'exercice de ce droit.

Il ne peut pas en être autrement quand on constate avec quelle énergie les autorités politiques fédérales s'opposent aux requêtes des détenus. 
En effet, il est étonnant qu'un gouvernement dit démocratique se refuse à adopter le principe que les jugements de cour ont une portée universelle en ce qui concerne la reconnaissance et le respect des droits des citoyens. Cela s'explique peut-être par le fait que les députés et ministres n'ont aucun respect pour les jugements qui mettent en cause leur soif de pouvoir. Lors de la reconnaissance du droit de vote des détenus aux élections provinciales d'Ontario par un tribunal ontarien, un ex-Solliciteur général du Canada et député, M. Robert Kaplan, déclarait à la Chambre des communes: «M. le Président, encore une fois, un tribunal inférieur s'est fondé sur la Charte des droits et libertés pour accorder aux détenus le droit de vote aux élections fédérales et provinciales, dans ce cas précis au niveau provincial... Je regrette beaucoup la tournure que prennent les événements et $\mathrm{j}$ 'implore les procureurs généraux d'interjeter appel de ces décisions rendues par des tribunaux inférieurs ${ }^{38}$."

Mais cette attitude vis-à-vis les jugements des tribunaux n'a pas cours que dans le dossier du droit de vote. Elle s'est manifestée dans d'autres domaines comme, par exemple, les fouilles et les tests d'urine obligatoires imposés aux détenus.

Il est toujours plus facile pour l'État d'étendre une restriction des droits que d'imposer des restrictions toutes nouvelles. Les brèches les plus aisées à effectuer sont celles qui concernent les détenus, puisque l'on peut sans peine justifier ces mesures en invoquant des raisons de sécurité publique. C'est ainsi que les détenus sont une cible privilégiée quand il s'agit de préparer le terrain.

Défendre les droits des détenus n'est pas une cause populaire, mais il faut bien se rendre compte que c'est non seulement défendre des personnes qui sont à la merci d'un pouvoir arbitraire, mais que c'est également défendre les droits et libertés de tous les membres de la société, les droits et libertés reconnus aux détenus s'appliquant finalement à tous et chacun.

L'histoire en général et l'histoire de la reconnaissance du droit de vote en particulier nous démontrent qu'il n'y a pas un droit, pas une liberté qui n'ait été gagné sans une lutte sociale contre le pouvoir étatique. Les droits ou libertés qui ne sont pas conquis demeurent des mots, sinon des rêves.

38. Débats des Communes, 10 août 1988, p. 18166. 